Vol. 1, No. 1, 2016

\title{
THE PRINCIPLES OF THE CYBER-PHYSICAL COMPONENTS' ORGANIZATION BASED ON THE METHODS OF THE MULTI-AGENT INTERACTION OF THE MOVING OBJECTS
}

\author{
Maxim Musiyenko, Iryna Zhuravska, Ivan Burlachenko and Oleksii Denysov \\ Petro Mohyla Black Sea State University (BSSU), 10, 68 Desantnykiv Str, Mykolaiv, 54003, Ukraine \\ Author e-mail: musienko2001@ukr.net
}

Submitted on 16.12.2015

(C) Musiyenko M., Zhuravska I., Burlachenko I., Denysov O., 2016

\begin{abstract}
In these article the mathematical model of multi-agent CFS is proposed for describe of behavior of moving objects, interacting under wireless communication link on 802.11 standard. It has shown that the more important criteria for CFS, consisting of mobile objects, is the stability of communications. The stable transmission rate is achieved by reducing the asymmetry of the link between the cyber-physical components. This will ensure the rhythmical loading of the computing board processors (CPU or / and GPU) on cyber object. The models of camera's matrices and computing boards for unmanned vehicles (UMV) are analyzed and proposed. The methods of avoidance the dropping of rate due to interference from the connecting to the ether of new access points, non-containing to CFS, are presented. The research and results contributes to the improving quality of transmission and processing data from wireless cyber-physical components.
\end{abstract}

Key words: cyber-physical components, multi-agent system (MAS), unmanned vehicles (UMV), wireless network, 802.11, video stream, computing board.

\section{INTRODUCTION}

Recently, it is taking place not only improvement the quality of the known types of interaction between the man and the physical world, but also the expansion the area of use of cyber-physical systems (CFS) in a variety of industrial, social, behavioral realizations of human needs. These multiple behavioral functions of CFS can't be implemented without the usage of mechanisms, androids, automated systems, both manned and is autonomous with algorithmic advance action. Topical is to achieve such an interaction between cyber-physical components, which will provide the necessary quality of functioning of the CFS [1].

At the same time, it is necessary to create and to implement algorithms of interaction between CFS objects such that fully utilize their technical capabilities (speed of information transmission and processing, range, payload motion and so on). This will force the cyber-physical components to work at the limit of their abilities and to interact into created network by the common rules [2].

Currently, the use of computer networks, working on prevalent protocols, is rather limited in CFS. However, the issues of network interaction between the cyberphysical components and interoperability CFS with the global Internet are the actual direction of research [3].

In the above work laid the foundation for further studies of various CFS, identified areas of scientific research and expected results. However, if before the majority of issues are resolved for CFS "man - a managed object" and "self-organizing systems", now the actual steel multi-agent systems. In such systems, management of multiple physical (self-contained or controlled) mobile units made by different people (cyber operators) through a variety of computing devices. Wireless connections, with the incessantly changing topology formed network, are used most frequently. Today fast dynamical CFS, which interacting via wireless communication protocols, are investigated the most insufficiently.

The purpose of this article is to use the methods of analysis of multi-agent systems (MAS) to describe the interaction of cyber-physical components connected by wireless communication standard 802.11, and to ensure stable data transfer rate across whole CFS area.

\section{MATHEMATICAL MODELS OF MULTI-AGENT CFS, COMPOSED OF MOVING OBJECTS}

Mesh-topology of CFS, which consists of moving objects, connected by wireless communication channels, provides non-periodic changes in relations between the different pairs of cyber-physical components. These pairs in said CFS may be:

a. One physical component (air, land, underwater, surface drones and other unmanned vehicle - UMV, equipped with sensors of physical processes) - another physical component.

b. Physical component - cyber-operator.

c. Cyber operator - cyber-operator. 
d. Cyber-operator - wireless access point (AP) cyber-operator.

e. Cyber-operator - wireless AP - telecommunication equipment - land Data Center.

Moving objects of CFS, united, for example, in the monitoring network, network delivery and other purposes as set $M M N=\left\{m_{1}, m_{2}, \ldots m_{n}\right\}$ is considered, where $m_{i}$ - is a node, which can be described using the direction of the motion vector $\bar{V}$, and has incidence matrix $I_{i, j}, i=j=N$. The subset of $M M N$ moving nodes, that is available for interaction, are shown in this matrix. The wireless transmission distances $D_{i}$ for data transfer from node $m_{i}$ have presented in the cells of the incidence matrix (1):

$$
I_{i j}=\left\{\begin{array}{c}
D_{i=j}, m_{i}=m_{j} ; \\
\left(\min \left\{D_{i}, D_{j}\right\}, \max \left\{D_{i}, D_{j}\right\}\right),(i, j) \in N ;
\end{array}\right.
$$

The distance $D_{i}(\mathrm{~m})$ and free space losses $L_{F S}(\mathrm{~dB})$ can be calculated as in (2) and as in (3) severally:

$$
\begin{gathered}
D_{i}=10{ }^{\left(\frac{L_{F S}-33}{20}-\lg F\right)} ; \\
L_{F S}=R x+G_{t r}-R x \text { Sens }-F_{S O M},
\end{gathered}
$$

where $F-$ is a frequency channel of transmitter node $(\mathrm{MHz}) ; \quad R x-$ is a power of transmitter node $(\mathrm{dBmW})$; $G_{t r}$ - the gain of transmitter node (dBi); RxSens - is a receiver sensitivity for defined transmission speed $(\mathrm{dBmW}) ; F_{S O M}-$ system operating margin $(\mathrm{dB})[4]$.

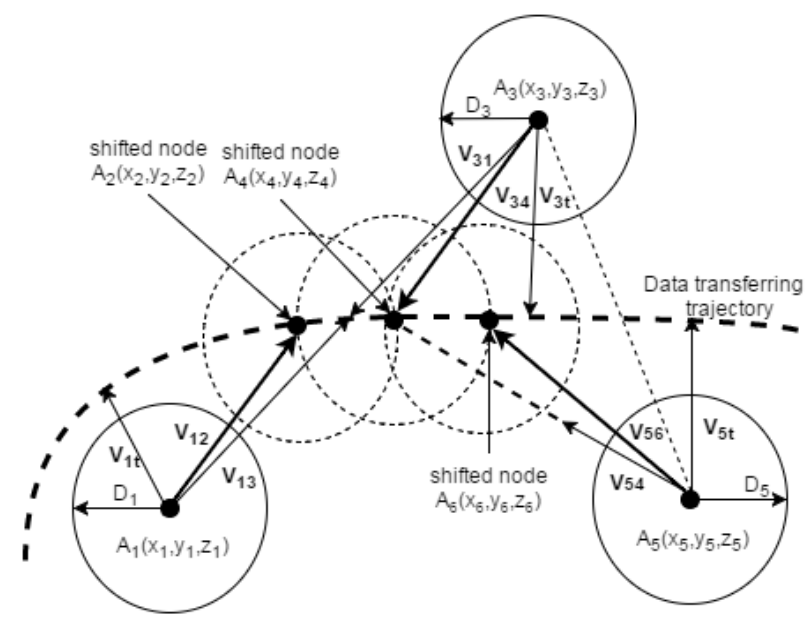

Fig. 1. Data transferring trajectory with moving nodes

Determining the distance of the signal $D_{l}$ allows to go to the next step of organizing the data transferring trajectory. Agent-based roles of nodes such as sender, repeater with checking the transferring success, repeater without checking the transferring success, receiver are defined. The $M M N$ node as transmitter is considered. The minimum value $\min \left\{D_{i}, D_{j}\right\}$ of a signal transmission distance provides data transferring between nodes of $I_{i, j}$ matrix in both directions. In cases of unidirectional data transfer, the maximum distance $\max \left\{D_{i}, D_{j}\right\}$ is used. The repeater without checking the transferring success as agent-based role of $M M N$ node with max distance has established.

In Fig. 1 the situation when nodes will move to trajectory and initiate data transfer is shown.

The node $A_{2}\left(x_{2}, x_{y 2}, z_{2}\right)$ shifted has sender's agent role. Considering displacement vector $\overline{V_{12}}=\overline{A_{1} A_{2}}$ in matrix form the shifting to area of data transferring trajectory (3) and rotation across $O X$ axis for location sender node on trajectory (4) can be determined [5]:

$$
\begin{gathered}
=\left[x_{1}, y_{1}, z_{1}, 1\right] \times\left|\begin{array}{cccc}
1 & 0 & 0 & 0 \\
0 & 1 & 0 & 0 \\
0 & 0 & 1 & 0 \\
D_{i} / \sin \alpha & D_{i}^{\prime} / \sin \beta & D_{i} / \sin \varphi & 1
\end{array}\right| . \\
{\left[x_{2}, y_{2}^{\prime}, z_{2}^{\prime}, 1\right]=} \\
=\left[x_{2}^{\prime}, y_{2}^{\prime}, z_{2}^{\prime}, 1\right] \times\left|\begin{array}{cccc}
1 & 0 & 0 & 0 \\
0 & \cos \beta & \sin \varphi & 0 \\
0 & -\sin \beta & \cos \varphi & 0 \\
0 & 0 & 0 & 1
\end{array}\right| .
\end{gathered}
$$

Let's motion of node $m_{i}$ is uniformly accelerated motion [6]. The time $T S_{i}$, while a node with index $i=1$ will move from point $A_{i}$ to point $A_{i+1}$ on data transferring trajectory, has shown in (6):

$$
T S_{i}=\sqrt{\frac{2 \cdot \sqrt{\left(x_{i}-x_{i+1}\right)^{2}+\left(y_{i}-y_{i+1}\right)^{2}+\left(z_{i}-z_{i+1}\right)^{2}}}{a}} .
$$

The sum value of $T_{t r}^{M M N}$ is estimated, as in (7). It describes the total value of the data transfer time and time of organizing the data transfer trajectory using $M M N$ nodes, which were in the arbitrary positions.

$$
T_{t r}^{M M N}=\left(\sum_{i=1}^{N-1}\left(T S_{i}\right)+T_{c t r}\right)+\left(T_{d t r}\right)^{q t r},
$$

where $T_{c t r}$ - time (ms) to send a certain amount $N_{c p}$ of communication messages with size $V_{c p}$ (measured in $\mathrm{KB}$ ) with data transmission speed $S_{t r}$ (Mbps) to establish a connection as in (8):

$$
T_{c t r}=\frac{N_{c p} \cdot V_{c p}}{S_{t r}}
$$

$q t r$ - specific coefficient of data packet transfer; $T_{d t r}$ transfer time (ms) of the data size $V_{d t r}(\mathrm{~KB})$, as in (9):

$$
T_{d t r}=\frac{V_{d t r}}{S_{t r} \cdot V_{c p}} .
$$


The message structure is based on an industry standard CAN, used in MAVLink protocol for transmitting data from the UAV, shown in Fig. 2 [7].

The communication message contains Package's system information (at head and trailer of message) and Payload fields. The head consists of the STX byte, which shows start of packet; LEN byte defines the length of payload; $S E Q$ is a number of packet in send sequence; SYS defines system identifier of MMN node; COMP defines the agent-based role identifier of MMN node; $M S G$ shows the data structure's type of payload. The Payload includes the data of the packet and depends on the $M S G$ byte value. The message trailer consists of the $C K A$ and $C K B$ indicates (low byte and high byte of packet's checksum severally).

For effective livelihoods, CFS is necessary to achieve the maximum symmetry of the communication channel between the Client (Station - STA) and an Access Point (AP). This requires equalize the flow rates are between upload and download. As a result, the difference between the signal-to-noise ratio (SNR) of AP and Client must tend to zero, as in (10):

$$
D F=\left(S N R_{S T A}-S N R_{A P}\right) \rightarrow 0 .
$$

Data-transfer mode with $D F<0$ (asymmetry of communication channels between the cyber-physical components) is critical for the CFS. In this case, any data rate from the Client to the AP (upload) will be slower than in the reverse direction (download), or there will be a loss of communication. In a result, cyber-operator will not able to manage UMV from their mobile device because the telemetry data will not be processed in adequate time, and UMV may to crash.

To achieve symmetry in the communication channels between the cyber-physical components it is needed to regulate the power $\mathrm{AP}$, so that $D F=0$.

Consider the relationships between the transmitter power $R x$ and receiver sensitivity RxSens for Client (STA) and AP respectively:

$$
\begin{aligned}
& S N R_{A P}=R x_{S T A}-\operatorname{RxSens}_{A P} ; \\
& S N R_{S T A}=R x_{A P}-R_{S} \text { Sens }_{S T A} .
\end{aligned}
$$

It's believed that the threshold of the background noise below the threshold sensitivity of the receivers Client and AP, as if the background noise level for AP and the Client is the same - it does not affect the symmetry of the channel [8].

Then transmitter power $R x$ is for $\mathrm{AP}$ and Client (STA) respectively:

$$
\begin{aligned}
& R x_{A P}=T x_{A P}+T x \text { Gain }_{A P}-L_{F S}+\text { RxGain }_{S T A} ; \\
& R x_{S T A}=T x_{S T A}+T x_{G a i n} \operatorname{sTA}-L_{F S}+R_{\text {G Gain }_{A P}} \text {; } \\
& \text { TxGain }_{A P}=R x \text { Gain }_{A P}=\text { Gain }_{A P} \text {; } \\
& \text { TxGain }_{S T A}=\text { RxGain }_{S T A}=\text { Gain }_{S T A} \text {, }
\end{aligned}
$$

where $T x_{A P}$ and $T x_{S T A}$ - the power of the transmitter at the AP and Client antenna port respectively;
TxGain $_{A P}$, RxGain $A P$ и TxGain ${ }_{S T A}$, RxGain RTA $_{-}$ antenna gains during transmitting or receiving for AP and Client respectively; $L_{F S}$ - losses in the communication channel between AP and Client (equal in both directions).

Given that the client devices are usually equipped with non-replaceable antenna, transmitter power and receiver sensitivity of the Client is indicated owing to the antenna immediately. Then the asymmetry of the channel is:

$$
\begin{gathered}
D F=\left(T x_{A P}-T x_{S T A}\right)- \\
-\left(\text { RxSens }_{S T A}-\text { RxSens }_{A P}\right) .
\end{gathered}
$$

The equation (10) - (13) shows that the asymmetry of the channel is independent of the antenna types on AP and Client (if not using multi-flow technology MIMO), but it depends on the difference between the transmitter power and receiver sensitivity of the cyber-physical components.

\section{THE EXPERIMENT PLANNING}

The half-duplex wireless connections are the multiple choice and interference vulnerable but they can pre-set before the communication session.

More often, many UMV-workers (not the headUMV) use boards, which haven't on-board Wi-Fi module. So, to optimize the interaction between CFS' objects is necessary to consider the question of choosing a computing board in detail. The additional modules have been installed there to create a channel of communication between the head UMV and / or cyberoperator. At the same time, mobile radio device, belonging to cyber-operator is integrate in device and can't replaced manually. Such a device could be a smartphone, netbook, laptop, remote control and similar.

Conversely, in the case of the communicate session is planned between the head UMV (from Parrot, DJI, MicroDrones Gmbh etc.), which has factory integrated wireless modules, and cyber-operator, it is necessary to choose the whole computer mobile devices, not a computing board only.

Consideration should be given the interaction between such pairs of wireless devices, which providing the telecommunications between UMV/UMV or UMV/cyber-operator, who managing UMV via any mobile device:

1) WiFi-adapters of UMV and mobile device operating Ad-hoc mode ("point-to-point").

2) UMV's Wi-Fi adapter (or mobile device's Wi-Fi adapter) and $\mathrm{AP}$, which operating Infrastructure mode ("Client-Server").

It is also necessary to be focused on such an obvious nuance as the principle of "three's a crowd", which becomes fundamental during the organization of cooperation within any wireless CFS. Thus, "three's a crowd" is not only a wireless Wi-Fi network of 
intersecting channels, but also the usual radio devices: USB-adapter for radio mouse, wireless keyboard, Bluetooth-headset and similar. All they can create significant interference interaction of CFS' objects.

A. Interaction Pair of UMV's Wi-Fi Adapter and Mobile Device

The largest number of cyber-physical systems use a modification of the Wi-Fi standard $802.11 \mathrm{~b} / \mathrm{g} / \mathrm{n}$. The desire of the CFS owner to use the latest standard among all the available is naturally.

This desire should be the fact that new standards support the highest speeds and should be backward compatible with previous versions. You can quite easily upgrade the wireless adapter in your computer or a laptop, but the modernization of other $\mathrm{Wi}-\mathrm{Fi}$ devices (e.g., smartphones, tablets and UMV) are not so simple.

To ensure a stable data rate is more appropriate to stick to one version of the standard for AP, and for mobile objects interacting with each other over Wi-Fi.

Absolutely, the current version of the standard $802.11 \mathrm{n}$ is actually today. However, the analysis of used devices, including identical models of mobile devices with the same hardware modules, has showed that compatibility is achieved at the exhaust standard $802.11 \mathrm{~b} / \mathrm{g}$ only.

The $802.11 \mathrm{~b} / \mathrm{g}$ standard isn't provided the full-duplex operation $[9,10]$. So, it is needed to pay attention on both the choice of wireless adapter models and the setting of mode these adapters for the organization of a half-duplex interaction between CFS objects.

Today the actual signal range in CFS is a distance of $50,100,250$ meters. In order to ensure such range, it is sufficient of standard power level up to $20 \mathrm{dBm}$. Such power level is corresponding to the level of $100 \mathrm{~mW}$ [11] and typical to the most commercial Wi-Fi adapter [12].

To ensure the long-range (up to $2 \mathrm{~km}$ in the absence of obstacles), you must install additional powerful Wi-Fi antennas of different configurations [13] and use of multi-flow technology (MIMO). The installation of additional antennas does not increase the power of the Wi-Fi module transmitter. However, such modernization can improve the radiation pattern of the module significantly. Thereby, the distance of cyber-physical components interaction is increased.

When we speak about the interaction of UMV and mobile devices, the role of the network's AP is performed the UMV Wi-Fi adapter. The control device (smartphone, laptop, remote control and similar) is connected with such AP $[14,15]$. Sometimes, the transmission of the broadcast traffic from UMV to several cyber-physical components is necessary. In this case, cyber-operator needs to connect to the UMV's AP in Bridge mode. Then it becomes possible to further the distribution of network, which is created by UMV, to other cyber-physical components [15].
B. Interaction Pair of UMV's Wi-Fi Adapter (of Mobile Device) - Access Point (AP)

The mode of auto-select the freest channel (Clear Channel Select) is set as default in the factory settings of the majority of Wi-Fi equipment models. However, the auto-change of channel can lead the breakage of the communication and to the loss of real-time video from UMV in a fast dynamic radio environment of the CFS work area. Therefore, it is appropriate to perform deactivation of the automatic channel selection mode in wireless equipment settings, used in the CFS, and manually set a Wi-Fi channel number, determined, as a result of studies.

Changing the wireless channel can help to increase the range and efficiency of interaction of CFS objects, especially if there is a neighboring network, being installed on the same channel or there interference from other devices. It is best to check the channels and to recommend a particular choice through such programsanalyzers as Vistumbler or InSSIDer [16].

It is appropriate to preserve the configuration of telecommunication devices (AP, router etc.) to a file for a multi-agent CFS with homogeneous objects. Then it is possible to transfer settings from one AP to the other APs within the CFS in case the expansion of CFS. This greatly simplifies and speeds up the wireless connection settings in case if the CFS will expand. Furthermore, it is possible to easily integrate new cyber-physical component into an existing mesh-topology and take into account changes of CFS behavior in formulas (1-9).

If you provide the management and configuration of cyber physical components using the remote / direct access to the built-in web interface, they will be configured by any cyber-operator, having an administrator right, from any mobile CFS object running iOS, Android or Windows Phone.

In our opinion, it is necessary to choose the wireless equipment that supports 802.1Q. This allows the sharing of physical network many logical (virtual) subnets (VLAN) means RADIUS. Standard RADIUS (Remote Authentication Dial-In User Service) provides crossroad of passwords from different authentication systems, allows mobile units, remaining members of the same VLAN (subnet unmanned aerial vehicles - UAV, subnet unmanned rovers, subnet other UMV, subnet cyber operators and other subnet of CFS objects), to navigate within the coverage of CFS and switch from one AP to another.

For rapid deployment CFS arbitrary and not only mesh-topology in a new area of action and with a large number of cyber-physical components (e.g., UAV monitoring network), the support of technology wireless backbones (WDS) by wireless telecommunications equipment should be useful. Using WDS-mode will integrate multiple wireless APs via communication channel without loss in the data rate. It is possible to allow simultaneous maintenance wireless clients on 
intermediate AP (in the "repeater" mode) or vice versa to deny access wireless clients (in the "bridge" mode).

C. Defining Characteristics of the Video Stream and Choice of Camera's Matrix for UMV

Given that the video stream transmission produces the largest load of channel link between UMV and cyber-object, you must define the minimum flow firstly, which the computing board of cyber object should be processing with the greatest speed to issue manage commands to UMV in order not to lose the last.

It should be noted, that it is necessary to correct the UMV movement path with the commands from the cyber-object by transmitting up to 30 managing commands per second in order to avoid the loss of UMV when you hover over it weapons or if there are obstacles in the way of UMV. Since, it is necessary to process at least four frames of the target image to issue manage commands to UMV. Therefore, a desired rate of the video stream is $120 \mathrm{fps}$ [17]. In addition, you will track objects, moving with high speed, thanks to high-speed video stream.

Firstly, you need to decide, what a video stream is. There are many video formats, which essentially boil down to one thing: a sequence of frames at a certain rate per second. Frame is image that being characterized through the resolution and the format (number of bits per pixel and interpretation: which bits of which correspond to the color component). Frame compression can be used inside the flow to reduce the amount of data transmitted. However, the screen shots are always decompressed to its original condition, when they are displayed. Restore of frames is needed also because the analytic algorithms also work with the uncompressed frames.

Thus, the video stream is characterized by frame rate, its format and image resolution. Importantly, the processing always at the same time has to deal with only one frame. That is why, they are processed sequentially. Furthermore, at the next processing, it is important to know how much time has passed since the previous frame. This value can be calculated from the frame rate, but more practical approach that is to support the frame with TimeStamp.

The first step is to prepare the frame before processing. As a rule, it is significantly reduced in size. The fact is that further processing will engage each pixel of image. Accordingly, the lower frame, the faster will be processed. Naturally, the jot of the information is lost in the frame during compression. But this is not critical, and even useful, for example, when motion is detected in the frame or the appearance of new objects. Objects are processing, mainly large enough to disappear of the frame during compression. However, all kinds of "noise" are associated with the quality of the camera, lighting, natural factors, will be reduced.

Changing the resolution can occur by combining several pixels of the original image into one. By way of association depends on the part of the information that will be saved. For example, the square 3 pixels $x 3$ pixels of the original image to be converted to a single pixel results. You can sum up all 9 pixels or can take a total of only 4 corner pixels and find the arithmetic mean, or you can take one of the central pixel value.

Another action at this stage is to change the format of image. Color images are not used generally, since it also increases the processing time of frame. For example, the additive color model $R G B 24$ comprises 3 bytes per pixel (24 bits). A conversion into 8-bit model $Y 8$, as in (14), leads to an image which occupies only a single byte per pixel, thus slightly yielding in a first information content:

$$
Y 8=(R+G+B) / 3,
$$

The result will be the same image, but in grayscale.

CMOSIS Comp. (Belgium) is engaged in the development and manufactures new types of solid-state optical sensors series CMV and CHR. A distinctive feature of CMOS-matrix CMOSIS company is having a high-resolution sensors which provide a high rate of frame reading [18].

Many of the matrices given above can be operated at a temperature of minus $30{ }^{\circ} \mathrm{C}$ to plus $70{ }^{\circ} \mathrm{C}$. This is an important parameter for outdoor CFS. Power dissipation of the sensor at full resolution and maximum frame rate can be dynamically controlled by reducing the frame rate.

Alternatively, it is possible to replace the CMOSmatrix of CMOSIS Comp. on, e.g., CMOS-matrix PYTHON-1300 color image sensor of Avnet, Inc. [19].

It should be noted, that there is the so-called "effect of jelly" (Rolling shutter) in CMOS-matrix, which are used for the production of cameras copter. This effect describes the delay of image of fast dynamic objects (which change their position a suddenly) on video, and as a result, geometric distortions or light strips are appeared on image. The way out of this situation is either to use CCD-sensor (which is not carried out by line, and full frame image transfer), or to use the hardware stabilization systems camcorders UMV or the imposition of certain digital filters already received video image.

The subtraction the low-frequency components of the image is executed during the reading the video signals of the exposed image from CCD-matrix and the converting them into digital form. Full frame is stored at the external memory. The brightness of the elements of this frame are the difference between the desired and the reference signal. Detected and location of detected objects in a picture image reading pace performs in parallel with the recording of the image in the memory.

It is used algorithms known as operators to work with windows to detect new objects. Window operators used window (some areas of the image) as an input. In this case, window is sized a 3 pixels x 3 pixels. The values of the pixels in this window are compared with a threshold value, which is floating and is calculated depending on 
the reading rate of the image. If there is a simultaneous excess of the threshold values of any three pixel's values in this window, then the found object will be fixed and in the vicinity of its area formed in measure $\mathrm{N} x \mathrm{M}$.

It is calculated the integrated brightness of the new object in each of the area with size $\mathrm{N}$ pixels $\mathrm{x} \mathrm{M}$ pixels in the pace of reading image shot from CCD-matrix. After the end of the field formation according with the values of integral luminosity it is sorted. The results of image processing images from CCD-matrix are:

- J luminance values of the brightest objects among being found. The brightness values are recorded in a block of 16 registers arranged in parallel.

- $\mathbf{J}$ values of the coordinates of the lower right corner of the area $\mathrm{N}$ pixels $\mathrm{x} \mathrm{M}$ pixels to $\mathrm{J}$ areas containing the most vivid found objects. The coordinate values are written in a block of $\mathbf{J}$ parallel registers.

All technologies of the video processing from the UMV require relatively high computing resources together with the requirements of minimizing dimensions of moving cyber-physical components.

\section{Choosing the Computing Board for UMV}

When you are engaged by programming both microcontrollers and programmable logic devices (PLDs), it is very important not to leave everything in theory, but implement ideas into practice immediately. You can do it in different ways, for example, do a variety of simulation as close to the real, or collect all in the layout. However, both of these methods have significant disadvantages:

- The work in the simulator may differ from that of the board.

- You can make a mistake and burn the controller / PLD or to connect something wrongly under assembling on the prototyping board. Then, you must make the long search for the mistake of trying to understand why the code does not work, although the problem is not somewhere in the code; In any case, it is required to build or to buy a programmer / computer interface.

A great "testing ground" for these issues is the evaluation board. It's such a board, where everything is connected properly and bred. Operation of "hardware" can be checked on the gauge demo program. Embedded systems debug and firmware allow to upload new firmware into the crystal in one motion. It remains only to experiment, without being distracted by extraneous factors.

Also, evaluation board serves as a convenient tool for rapid prototyping and running knots, checking the ideas and methods.

It makes sense - at first to assemble at demo board, to verify that the idea works, adjust as needed, and then debugged code already to use in a real project. In developing the device so, you can save a lot of time.

To work with data from CMOS-matrices followed a video stream processing is proposed to use SoC. The reasons for the choice is simple: the processing of the video stream with $120 \mathrm{fps}$ and above, the microprocessor will not be able to produce. For working out of the solutions, it is proposed to take evaluation board Zedboard. The functionality of this board presented in Fig. 3 [20]. Characteristics of PLD SoC are listed Table I [21].

On the evaluation board set SoC Z-7020.

For working off the SDR (Software Defined Radio) solutions you can also use board Zedboard, just putting radio module Zynq-7000 AP SoC / AD9361 with following characteristics [22]:

- Combines Avnet Zedboard with Analog Devices AD9361 integrated RF Agile Transceiver.

- RF $2 \times 2$ transceiver with integrated 12-bit DACs and ADCs.

- Band: factory-tuned for optimal performance at 2400 - $2500 \mathrm{MHz}$.

- User-modifiable to other RF center-frequencies from $70 \mathrm{MHz}$ to $6.0 \mathrm{GHz}$.

- Tunable channel bandwidth at the data converter: $<200 \mathrm{kHz}$ to $56 \mathrm{MHz}$.

- Powered from single FMC connector.

- Supports MIMO radio, with less than 1 sample sync on both ADC and DAC.

- Includes schematics, layout, BOM, HDL, Linux drivers and application software.

- Supports add on cards for spectrum specific designs (PA, LNA etc.).

- SPI access for all device registers.

- Optional MathWorks tools support for communications system design.

Due to such construction SDR, you can implement functions FHSS (Frequency-Hopping Spread Spectrum), protected noise coding, data encryption, and so on.

Thus, by the usage of the Zedboard you can debug independently video processing (on the matrix of CMOSIS Comp.) with 120 fps or above and a job with a SDR.

Thereafter, it should be decided how to build the system as a whole, depending on the load on ARM9 processor, and the number of occupied logic PLD. In the future for high-speed data transmission it is possible to use high-speed transceivers, which are presented in the "older" models SoC and provide data transmission speed up to 3.125 Gbps.

Zedboard has shortage of the block of memory to handle high-resolution video. But, due to the scalability of the algorithm processing, you can first check on the low resolution video image of like $640 \times 480$ or less.

The usage of PLD is an important advantage, such as the ability processing of parallel processes. PLD can collect information from all sensors in parallel and process of data at the same time. The results of the processing can to use to management of multiple servo through drivers.

It should be noted, that Zedboard has integrated HDMI-transmitter only. Therefore, to be able to receive 
on Zedboard video via HDMI, you can use the board FMC-HDMI-CAM FMC [23].

Summary $A-D$, before you start CFS with wireless communication protocols, you must:

1) Conduct a study radio environment in the whole CFS area by means of one of the software or hardware wireless network analyzers (only selected standard).

2) Set the interaction of CFS objects on the most uninhabited channel (with the fewest quantity of SSID).

3) Adjust the power limitation AP transmitter in accordance with the calculated parameters of the channel asymmetry based on SNR of each pair interacting cyberphysical components.

4) Define characteristics of the video stream and choose of camera's matrix for UMV.

5) Choose the computing board for head UMV.

\section{RESEARCH RESULTS}

In the experiment, the data rate determination was carried out:

a. From a moving object on the ground to motionless cyber-object (with devices based on the operating system Windows 8.1), which is in optical line-of-sight, - by means of built-in operating system tools.

b. Between two moving cyber-physical components (laptops, tablets, smartphones), which communicate with each other using APs and are managed by people, - by means of built-in operating system tools.

c. Between two moving cyber-physical components, which communicate with each other directly and are managed by people - by means of the program BArtWell Wi-Fi File Sender, versions of which are available for Android, Windows Mobile and PC [24].

\section{E. Analysis of Interference from Neighboring Wi-Fi} Networks and Selection of Channel Link Number

We have studied the dynamics of the interaction of the two most common Wi-Fi adapter Ralink RT3290 $802.11 \mathrm{~b} / \mathrm{g} / \mathrm{n}$ [25] built-in the laptop model ASUS X501U running Windows 8.1 SL. In this mode, one of the adapters works as an AP.

The problem is that if the AP operates as "only 802.11n", other Wi-Fi adapter Ralink RT3290 will not see this AP that was created on the same adapter, even within the coverage area.

It was noted that the decision to limit the speed of data transmission in a wireless CFS at the level below than the maximum of mode "only $802.11 n$ " is irrational only when the first consideration. However, it is justified after the following arguments.

Therefore, the highest attainable experimental result of used Wi-Fi adapter Ralink RT3290 is at the level of 80-90 Mbps, despite of the maximum connection speed is allowed up $150 \mathrm{Mbps}$. At the same time, in operating condition the 802.11n, AP uses two frequency bands, which means that it will need more power. Given, that AP is created on UMV, it leads to unwarrantable the overspending of battery life.
To do less energy consumption, therefore it is advisable to limit the speed of interaction of CFS objects uniform level of standard $802.11 \mathrm{~g}$. If you set the mode of operating conditions " 802.11 bgn mixed", the link will be "802.11g only" protocol with the inherent speed of up to $54 \mathrm{Mbps}$ (actually $25 \mathrm{Mbps}$ ). But, this data rate is enough to interact the cyber-physical components, even for implementation FPV (First Person View) mode on UAV.

Updating the adapter drivers [26] do not solves this problem.

Working on the $5 \mathrm{GHz}$ frequency is stayed seeing as the band with the more demanding the presence of the optical line of sight. If it is put the task of reducing the time of life (data transfer) CFS or the usage of less energy-intensive (thereby, lighter) batteries, it will be necessary to consider usage the equipment on IEEE 802.11ac standard in these tasks. In this case, the interaction of cyber-physical objects will be with higher data rate (with achievable experimental results in $290 \mathrm{Mbps}$ ) at a frequency of $5 \mathrm{GHz}$ [27].

Thus, at the setting Wi-Fi adapter Ralink RT3290 $802.11 \mathrm{~b} / \mathrm{g} / \mathrm{n}$ it has previously selected the channels \# 1-13, corresponding to the $2.4 \mathrm{GHz}$ frequency band (Fig. 4).

By means of the program InSSIDer 4 [16] it has analyzed the radio environments on the selected channels (Fig. 5), has tested the quality of communication within the target Wi-Fi network, which has SSID "u_r_not_prepeared" (Fig. 6).

The result showed the unsatisfactory performance of the communication channel when the response of the echo packet was about 2 seconds. These characteristics do not allow to view and process the transmitted video stream data real-time.

Also, it decided to narrow bandwidth of communication channel to improve the quality of communication. Typically, this is done using nonoverlapping channels \# 1, 6, 11 in $2.4 \mathrm{GHz}$ band.

However, among the non-overlapping channels $2.4 \mathrm{GHz}$ band channel \# 6 is the most common. This channel is set the default for most switching Wi-Fi equipment [28]. In Fig. 5 also is shown that there are five networks with a power of a signal approximate to the target network with SSID " $u \_r \_n o t \_p r e p e a r e d "$ signal level, in displayed part in the monitoring area of the program InSSIDer. Therefore, configure CFS objects on this channel does not make sense.

Given, that most UAV Wi-Fi modules is configured to far channels \# 11-13 of the $2.4 \mathrm{GHz}$ band [29], the following experiment to analyze the radio environment was conducted using $\mathrm{Wi}-\mathrm{Fi}$ analyzer program InSSIDer 4 for bandwidth, which is limited by channels \# 10-13 (Fig. 7).

It was found that the target network with SSID " $u \_r \_n o t \_p r e p e a r e d "$ (Fig. 8) has the most power ($69 \mathrm{dBm})$ in the selected channel \# 13 as compared with only two neighboring network $(-96 \mathrm{dBm})$, which work in this channel. 
However, this positive radio situation and the nonexistence of noise in communication channel between CFS objects improved the quality of communication slightly (Fig. 9). Instead of $2 \mathrm{~s}$ in the previous experiment, when the new configuration properties cyber-physical component has become effective, response time of echo packet dropped just twice and amounted to about $1 \mathrm{~s}$. Such connection speed is insufficient to speed dynamical processes and processing video stream real-time.

In such a situation, it is entirely justified to conclude that the choice of the standard and of unloaded communication channel will not lead to further improving the quality of the communication channel between wireless CFS objects.

\section{F. Achieving of Communication Channels Symmetry}

We has researched the symmetry of the communication channel between pair AP-Client to improve the quality of the communication channel. We should verify the initial setting of transmitter signal power to outdoor applications access point D-Link DAP3310 (Fig. 10). It can be seen that AP has the maximum transmitting signal power in the experiment.

According to Data Sheet, $100 \%$ transmitter outputs the power of the DAP-3310 is $20 \mathrm{dBm}$ at $6-36,48$, $54 \mathrm{Mbps}$. Receiver sensitivity is $-74 \pm 2 \mathrm{dBm}$ at $54 \mathrm{Mbps}$ [30]. The Wi-Fi adapter Ralink RT3290 802.11b/g/n [25], which is built-in laptop models ASUS X501U, has characteristics:

- Output Power 802.11g: $14 \mathrm{dBm}$ +/- $1.5 \mathrm{dBm}$;

- Receive Sensitivity $802.11 \mathrm{~g}$ : less than $-70 \mathrm{dBm}$ (54 Mbps).

For the setting power of a signal in the AP $(100 \mathrm{~mW}=20 \mathrm{dBm})$ and the Client $(18-35 \mathrm{~mW} \sim=12.5-15.5 \mathrm{dBm})$ power difference is minus $4.5-7.5 \mathrm{~dB}$.

The calculation of the maximum of the communication channel asymmetry has fulfilled the notebook ASUS and AP D-Link DAP-3310 with 802.11g@54Mbps:

$$
\begin{aligned}
D F & =(12.5-20)-(70-72)= \\
& =-7.5+2=-5.5 \mathrm{~dB} .
\end{aligned}
$$

As in (15), problems may occur in interaction between CFS objects, and the AP is fault in that.

To obtain a stable communication, the AP signal power must be reduced. Minimum asymmetry of the communication channel, which can be achieved, is as in (16):

$$
\begin{aligned}
& D F=(12.5-50 \% * 20)-(70-72)= \\
& \quad=-2.5+2==-0.5 \mathrm{~dB} .
\end{aligned}
$$

When the level Transmit Power $50 \%$ has set in the AP D-Link DAP-3310 web interface, it makes sense to check the ping between the studied cyber-physical components (Fig. 11). It can be seen that the response time of the echo packet decreased almost an order of magnitude.
In the result of the latter experiment, the communication quality in the wireless channel is significantly improved.

\section{G. Determination of Wi-Fi Speed Dependence from} the Distance

There was investigated some models of SOHO- and SMB-segment wireless equipment to outdoor applications operated in AP mode (production of TP-Link, Huawei-3Com and D-Link companies).

Theoretical speed declared at $54 \mathrm{Mbps}$, in the real conditions falls to $12 \mathrm{Mbps}$ at a distance of $50 \mathrm{~m}$ (Fig. 12, line 1). This is so due to a rather complex radio environment in the $2.4 \mathrm{GHz}$ frequency because this band belongs to the unlicensed ISM-band. The reason for the speed reduction is also interference the signals from other networks on the same standard. Communication speed may drop down to 1-2 Mbps at the same distance for moving objects [9]. Accordingly, the rate of $54 \mathrm{Mbps}$ can be ensured at the receiver sensitivity $-74 \pm 2 \mathrm{dBm}$. If the receiver sensitivity is about $-90 \mathrm{dBm}$, the rate drops to 2-5 Mbps [31].

According to the company Cisco information, data transmission on standard $802.11 \mathrm{a} / \mathrm{b} / \mathrm{g}$ can be achieved with higher levels of speed (Fig. 12, line 3) on equipment Cisco Aironet Series 1130 AG [32]. However, the stepped change of the speed of data transmission in different CFS areas will lead to uneven loading of computing board processors and, as a result, in overheating and possible hang-up of cyber operator devices with consequent data loss from the physical components (sensors, camera etc.).

For UAVs, the power signal transmitter of standard Wi-Fi devices (not more than $20 \mathrm{dBm} / 100 \mathrm{~mW}$ [11]) provides a distance of equipment activity to $200 \mathrm{~m}$ without additional amplification. The enhancing interaction zone of cyber-physical components up to $400 \mathrm{~m}$ needs more power signal (relevant $25 \mathrm{dBm} / 316 \mathrm{~mW}$ or more). However, in Ukraine, this requires permission from the National Commission for the State Regulation of Communications and Informatization (NCCIR of Ukraine).

The increase of power signal transmitter above the computation, referred previously, is necessary to transfer the video stream real-time in HD-quality at a distance of $700 \mathrm{~m}$ on the Wi-Fi. There are such capabilities, e.g., in quadrocopter DJI Phantom 2 Vision, which can be used as a head of the UAV "flock" drones. The usual distance of UAV's equipment does not exceed $50 \mathrm{~m}$ (e.g., quadrocopter Parrot AR.Drone 2.0 Elite Edition Sand). Its receiver supports $\mathrm{Wi}-\mathrm{Fi} 802.11 \mathrm{~b} / \mathrm{g} / \mathrm{n}$ and provides managing by cyber operator mobile device based on iOS or Android that supports Wi-Fi 802.11b/g/n.

The data rate, depending on the distance in meters, is shown in Fig. 12, line 2. This dependence was obtained, as a result of the experiment, described in paragraphs $A$ and $B$ this section of article. The data rate has stabilized at a value of $24 \mathrm{Mbps}$ over the entire CFS area (up $200 \mathrm{~m}$ ) for the current wireless network topology. 
The border of the experiment area was determined accordance with significant interference from highvoltage transmission power line. These noises have caused a sharp drop in the transmission rate and total interruption of the communication channel between cyber physical components when the distance between them exceeded more than $200 \mathrm{~m}$. Thus, the cut line 3 to zero in Fig. 12 does not show the boundary possibilities standard $802.11 \mathrm{~g}$. To resume the communication channel it is necessary to make a new research of radio environment by means of software or hardware wireless analyzer (e.g., InSSIDer) and to redefine the parameters of the link between the CFS objects. Similar new researches will be necessary also, if the new APs start operating on the same communication channel, with comparable power signal level in the vicinity to CFS zone activity.

\section{CONCLUSIONS}

1) In this paper, we proposed a mathematical model of multi-agent wireless CFS consisting of moving objects. This model adequately describes the interaction of cyber-physical components, which are combined into a monitoring network, delivery network or network of other destination. Conducted experiments has confirmed the significant difference between the theoretical performance $\mathrm{Wi}-\mathrm{Fi}$ equipment about data rate and communication range from the real values when CFS operates in the territory of the city (settlement) with a noisy radio environment.

2) By analyzing the modes of wireless data transmission between pairs of cyber-physical components, we have shown that the more important criteria for CFS, consisting of mobile objects, is the stability of communications, regardless of achievable speed maximum in according with the standard.

3) By reducing the asymmetry of the link between the cyber-physical components, we have achieved the stable transmission rate of $24 \mathrm{Mbps}$. This will ensure the rhythmical loading of the computing board processors (CPU or / and GPU) on cyber object. The achieved data rate is sufficient for the qualitative processing of the video signal transmitted from the UMV to cyber object.

4) We have proved that it is possible to avoid the dropping of rate due to interference from the connecting to the ether of new access points, non-containing to CFS. This is achieved by redefining radio environment in the CFS work area and changing characteristics of cyberphysical components. At the same time, you can avoid falling rate when CFS moving objects are entering in areas of high electromagnetic noise or optical line-ofsight is lost between the cyber-physical components.

5) It is possible the overcoming of the limit distance in wireless CFS by using additional special configurations of antennas with different gains, and also by means of manage the signal power on cyber-physical component of all CFS levels.

\section{REFERENCES}

[1] A. Melnyk, (2014), Cyber-Physical Systems: The Problems of Creation And Directions of Development, Visnyk of the National University "Lviv Polytechnic". Computer systems and networks, no. 806, pp. 154-161.

[2] A. Casavola, E. Garone, and F. Tedesco, (2014, Aug.), A Distributed Multi-Agent Command Governor Strategy for the Coordination of Networked Interconnected Systems, IEEE Trans. on Automatic Control. A Publ. of the IEEE. Control Systems Society, vol. 59, no. 8. [Online]. Available: http://www. ieeecss.org/sites/ieeecss.org/files/CSS-Digest-August2014.pdf.

[3] E. A. Lee, Cyber-Physical Systems - Are Computing Foundations Adequate? In Position Paper for NSF Workshop On Cyber-Physical Systems: Research Motivation, Techniques and Roadmap, Austin, TX, USA, October 16-17, 2006. [Online]. Available: http://ptolemy.eecs.berkeley.edu/publications/ papers/06/CPSPositionPaper/Lee_CPS_PositionPaper.pdf.

[4] A. V. Proletarskiy, I. V. Baskakov and D. N. Chirkov, Wireless Wi-Fi networks, in Plastics, Moscow, Russia: BINOM, 2007, 142 p.

[5] D. Hearn and M. P. Baker, Computer Graphics with OpenGL, 3rd ed., Upper Saddle River, NJ : Person Prentice-Hall, 2004, 857 p.; ISBN: 0-13-015390-7.

[6] G. S. Landsberg, "V.1. Mechanics. Heat. Molecular Physic" in Elementary physics, Moscow, Russia: Nauka, 1985, 606 p.

[7] W. Voss, "A Comprehensible Guide to Controller Area Network", Copperhill Technologies Corporation, Amherst, Massachusetts, 2005, p. 34.

[8] A. Bandurian. (2014, Feb. 16). Wi-Fi, the unobvious and overlooked: Power. In Technical Blog. [Online]. Available: https://arsenb.wordpress.com/2014/02/16/wi-fi-the-unobviousand-overlooked-details-part-1/.

[9] I. A. Kubasov, P. N. Serdyukov and oth. (2013, Feb.). Estimation of communications capacity and range when employing Wi-Fi equipment in real urban environment. Special equipment. [Online]. Available: http://www.bnti.ru/showart.asp?aid= 1018\&lvl=04.01.01.03.\&p=1

[10] Wi-Fi (Wireless Fidelity) - wireless standard. (2015, Aug. 17). TAdviser Portal. (Technologies + Adviser) [Online]. Available: http://www.tadviser.ru/index.php/Статья:Wi-Fi_(Wireless_ Fidelity)_-_стандарт_беспроводной_связи_802.11

[11] $\mathrm{dBm}$ to $\mathrm{mW}$ conversion. (2015). Online Scientific and Engineering Resource. [Online]. Available: http://www. rapidtables. com/convert/power/dBm_to_mW.htm.

[12] Wi-Fi Adapters (2015, Nov.). Internet-shop "STYLUS". [Online]. Available: http://network.stylus.com.ua/ru/products/ wifi_adapters/index.html.

[13] E. Geier. (2012, Aug. 28). How to Extend Your Wi-Fi Network. PCWorld homepage. [Online]. Available: http://www.pcworld. com/article/261495/how_to_extend_your_wi_fi_network.html.

[14] The U. S. military wants to distribute Wi-Fi with drones. (2014, Apr. 18). [Online]. Available: http://m.kaskus.co.id/thread/ 5350c9f2f9ca174c398b473c/the-us-military-wants-to-distribute-wi-fiwith--drones/?ref=postlist-54\&med=recommended_or_you.

[15] The WiFi-amplifier to AR.Drone 2 and other small items. (2015, Apr. 12). In The art of system administration. [Online]. Available: http://macrodmin.ru/2015/04/wifi-ar-drone-2.

[16] Troubleshoot Your Wi-Fi with InSSIDer. (2015). In MetaGeek.Com. [Online]. Available: http://www.inssider.com/.

[17] V. Slusar, "Data transmission on board the UAV: NATO standards", Electronics: Science, Technology, Business. no. 3, pp. 80-86, 2010.

[18] CMOSIS' standard image sensors. (2015). [Online]. Available: http://www.cmosis.com/.

[19] ON Semiconductor PYTHON-1300-C Camera Module. (2014). Avnet, Inc. [Online]. Available: http://zedboard.org/sites/default/ files/product_briefs/PB-AES-CAM-ON-P1300C-G-v2-web.pdf

[20] Zynq 7010 / 7020 SOM (System-On Module). Version 1.4. (2015, Mar. 2). [Online]. Available: http://zedboard. org/sites/default/ iles/documentations/PicoZed_7010_7020_User_ Guide_v1_4.pdf. 
[21] Zynq-7000 All Programmable SoC Overview. DS190. Version 1.8. (2015, May 27). [Online]. Available: http://www.xilinx.com/ support/documentation/data_sheets/ds190-Zynq-7000Overview.pdf.

[22] Zynq-7000 AP SoC / AD9361 Software Defined Radio Evaluation Kit. (2013). In Avnet Product Brief. [Online]. Available: $\quad$ http://www.em.avnet.com/en-us/design/drc/ Documents/Designed\%20By\%20Avnet/PB-AES-ZSDR2-ADIG-V1d.pdf.

[23] HDMI Input/Output FMC module with Camera Interface. (2015). In Avnet Community Forum. [Online]. Available: http://www.em.avnet.com/en-us/design/drc/Pages/HDMI-InputOutput-FMC-module-with-Camera-Interface.aspx.

[24] A. Bazhanov. (2014, Dec.). Wi-Fi File Sender v. 4.10 [Online]. Available: http://bartwell.ru/soft/android/wififilesender/.

[25] AzureWave AW-NB087H Ralink RT3290 Chipset IEEE 802.11 b/g/n Wi-Fi Card: Features. (2014, Dec.). [Online]. Available: http://www.amazon.com/AzureWave-AW-NB087H-Chipset150Mbps-Bluetooth/dp/B00EZ01OQU.

[26] Device types - Network Adapters - Ralink Technology, Corp. Ralink RT3290 802.11bgn 1x1 Wi-Fi Adapter Drivers Download. (2012, Apr. 12). In DriverMax [Online]. Available: http://www.drivermax.com/driver-download/0/Network+ Adapters/Ralink+Technology, +Corp./Ralink+RT3290+802.11bgn+1 x1+Wi-Fi+Adapter

[27] 802.11ac: The Fifth Generation of Wi-Fi. In Technical White Paper. Cisco Systems Incorp., San Jose, CA, USA, March, 2014. [Online]. Available: https://www.cisco.com/c/en/us/ products/collateral/wireless/aironet-3600series/white_paper_c11-713103.pdf.

[28] A. V. Polynkin, and H. T. Le, "Analysis of characteristics of UAV communication", Journal Tula State University. Technical Science, no. 7-2, pp. 98-107, 2013. [Online]. Available: http://cyberleninka.ru/article/n/issledovanie-harakteristikradiokanala-svyazi-s-bespilotnymi-letatelnymi-apparatami.pdf.

[29] Parrot Bebop + Skycontroller - quadrocopter with navigation and camera. (2015, Oct.). In Medgadgets: First blog-shop smart electronics. [Online]. Available: http://medgadgets.ru/shop/parrot-bebop-skycontroller.html.

[30] Продукты и решения - Беспроводное оборудование - DAP3310. (2015). In D-Link (Europe) Ltd. [Online]. Available: http://www.dlink.ru/ru/products/2/1790_b.html.

[31] E. Geier. "7 Tips to Increase Wi-Fi Performance". (March 8, 2012). In Wi-Fi planet homepage. [Online]. Available: http://www.wi-fiplanet.com/tutorials/7-tips-to-increase-wi-fiperformance.html.

[32] Cisco Aironet 1130AG IEEE $802.11 \mathrm{a} / \mathrm{b} / \mathrm{g}$ Access Point. Data Sheet. (2009). [Online]. Available: http://www.cisco.com/c/en/us/ products/collateral/wireless/aironet-1130-ag-series/product_ data_sheet0900aecd801b9058.pdf.

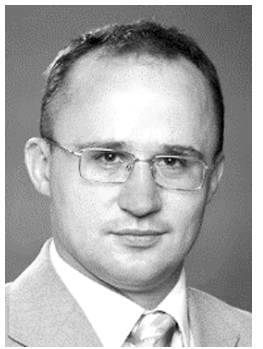

Maxim Musiyenko received his higher education in "Radiotechnics" from Cherkasy Engineering and Technological Institute, in 1998. In 2001, he has defended his Ph.D. thesis, in 2006 - Doctor of Science in computer systems and components (at the Odessa National Polytechnic University). In 2004, he received the academic rank of Assistant Professor, in 2007 - Professor. Since 2011, he is the Head of Department of Information Technologies (IT) and Software Systems at the Petro Mohyla Black Sea State University (BSSU), Mykolaiv, Ukraine. Since 2015, he is the Dean of the Computer Science Faculty (CSF) at the Petro Mohyla BSSU.

$\mathrm{He}$ is the author of over 500 scientific works, including four books, and holds more than 200 patents.

He was the Cabinet of Ministers of Ukraine Prize Laureate (2003), Grantee President of Ukraine for young scientists (2006, 2008).
His research interest includes the computer engineering in the field of embedded navigation and communication systems; sensor electronics.

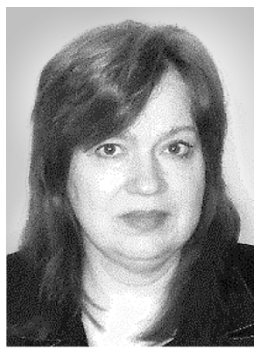

Iryna Zhuravska received her higher education from Mykolaiv Shipbuilding Institute (now Admiral Makarov National University of Shipbuilding) in 1985. She has defended her theses at the Institute of Electrodynamics of the National Academy of Sciences of Ukraine (Kyiv) and received the Candidate of Technic al Sciences degree in 1994. In 2007, she received the Ph.D. degree in semi-conductor electric power converters and the academic rank Associate Professor of Information Technologies and Documentation.

Since 2008, she is an Associate Professor with the Department of IT and Software Systems of CSF at the Petro Mohyla BSSU, Mykolaiv, Ukraine. She researches the design, implementation and information security of computer network systems, including SCS-networks, Wireless, PowerLine and other network infrastructure. She is the author of one book, more than 50 articles and conference abstracts, holds 4 patents.

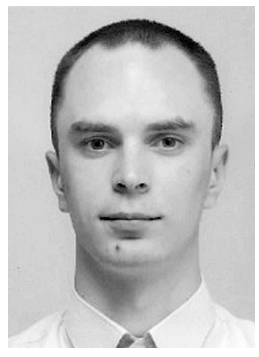

Ivan Burlachenko received the B.S. degree in computer science, in 2008 and the M.S. degree in decision support systems and methods from Petro Mohyla BSSU, Mykolaiv, Ukraine, in 2010. He is pursuing the Ph.D. degree in information technologies at Petro Mohyla BSSU, Mykolaiv, Ukraine.

After graduation from Petro Mohyla BSSU, he spent several years working as a Java developer and JavaScript developer. Since 2010, he is a Research Assistant with the Department of IT and Software Systems of CSF at the Petro Mohyla BSSU, Mykolaiv, Ukraine.

$\mathrm{He}$ is the author of near 20 articles and conference abstracts. His research interest includes artificial intelligence, multi-agent systems, machine learning, data mining, wireless sensors networks with low energy consumption. He is getting involved in applying trend technologies (according to PyPL) to research work.

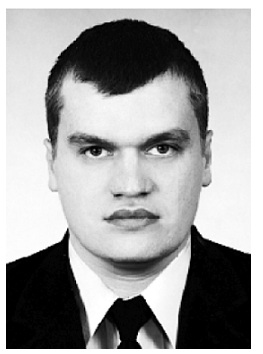

Oleksii Denysov received his higher education in "Radiotechnics" from National Technical University of Ukraine "Kyiv Polytechnic Institute", in 2005.

Since 2001, he has been an electronics engineer-designer with the Arsenal special instrument engineering enterprise, JSC SEA Co. Ltd, UTAS Co. (all are located in Kyiv). He is the author of near 10 articles and conference abstracts, holds one patent. His research interest includes the MCU and FPGA structural, functional and electrical circuit development and software design, development of DSPalgorithms. He composes technical and review articles on programming for the FPGA company's site and publication in the print media.

In addition, he is professionally programmer on $\mathrm{C} / \mathrm{C}++$ for STM32 and Atmel AVR (8bit) microcontrollers, on VHDL/Verilog for FPGA/CPLD (Altera, Xilinx and Lattice), developer of mathematical models in Matlab for verification of digital and analog circuit technique. 